\title{
Erratum to: Adrenal fatigue does not exist: a systematic review
}

Flavio A. Cadegiani and Claudio E. Kater ${ }^{*}$

\section{Erratum}

Following publication of the original version of this article in BMC Endocrine Disorders [1] it was brought to our attention that the comment section for row 12 in Table 1 should be blank. Please find the corrected table below.

Received: 28 August 2016 Accepted: 9 September 2016

Published online: 16 November 2016

\section{Reference}

1. Cadegiani FA, Katerl CE. Adrenal fatigue does not exist: a systematic review. BMC Endocrine Disorders. 2016;16:48.

* Correspondence: kater@unifesp.br

Adrenal and Hypertension Unit, Division of Endocrinology and Metabolism, Department of Medicine, Escola Paulista de Medicina, Universidade Federal de São Paulo (EPM/UNIFESP), R. Pedro de Toledo 781- 13th floor, 04039-032 São Paulo, SP, Brazil
Submit your next manuscript to BioMed Central and we will help you at every step:

- We accept pre-submission inquiries

- Our selector tool helps you to find the most relevant journal

- We provide round the clock customer support

- Convenient online submission

- Thorough peer review

- Inclusion in PubMed and all major indexing services

- Maximum visibility for your research

Submit your manuscript at www.biomedcentral.com/submit

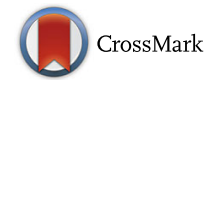


Table 1 Summary of selected studies

\begin{tabular}{|c|c|c|c|c|c|c|c|}
\hline First author \& reference & $\begin{array}{l}\text { Year of } \\
\text { publication }\end{array}$ & $\begin{array}{l}\text { Number } \\
\text { of } \\
\text { patients }\end{array}$ & Population & Questionnaire & Tests & Results & Comments \\
\hline McLennan [21] & 2016 & 257 & $H / B$ & Maslach & $\mathrm{HCC}$ & $\mathrm{Nl}$ & \\
\hline Schmidt [69] (part 1) & 2016 & 265 & $\begin{array}{l}\text { Breast } \\
\text { Cancer }\end{array}$ & FAQ & DAC, CAR, SCR, NSC & $\begin{array}{l}\uparrow N S C, \uparrow A \cup C, N I C A R, N I D A C, \\
\downarrow S C R\end{array}$ & $\begin{array}{l}\text { Pts. mostly on chemotherapy (ChTx); schemes not } \\
\text { specified. No controls, only correlation between fatigue } \\
\text { levels and tests. ChTx related to worse fatigue. Initial tests } \\
\text { performed during, w/ or w/o ChTx. }\end{array}$ \\
\hline Schmidt [69] (part 2) & 2016 & 265 & $\begin{array}{l}\text { Breast } \\
\text { Cancer }\end{array}$ & FAQ & DAC, CAR, SCR, NSC & $\begin{array}{l}\uparrow A \cup C, \uparrow C A R, \uparrow N S C, \uparrow S C R, N I \\
\text { DAC }\end{array}$ & $\begin{array}{l}\text { Second test of the study, performed after } 14 \text { weeks of } \\
\text { procedures. }\end{array}$ \\
\hline Sjors [25] & 2015 & 220 & $H / B$ & SMBQ & DAC, CAR & $\downarrow C A R, \uparrow D A C$ & $\begin{array}{l}\text { Results normalized after adjustment of anti-depressive use; } \\
\text { SCR results not provided }\end{array}$ \\
\hline Oosterholt [28] & 2015 & 91 & $H / B$ & Maslach & $\begin{array}{l}\text { DAC, CAR, SCR, AUC, } \\
\text { CAR 60min }\end{array}$ & $\begin{array}{l}\text { NI SCR, } \downarrow C A R, N I A U C, N I C A R \\
60 \mathrm{~min}, \downarrow D A C\end{array}$ & Control of variations did not change results \\
\hline De Vente [22] & 2015 & 95 & $H / B$ & $\begin{array}{l}\text { Acute } \\
\text { Psychosocial } \\
\text { Stressor } \\
\text { diagnosis }\end{array}$ & MSC, MST & $\mathrm{NI}$ MSC, NI MST & $\begin{array}{l}\text { MST: NI (women), reduced (men); MSC: NI (men), reduced } \\
\text { (women). Mental arithmetic and public speech stressors } \\
\text { also performed }\end{array}$ \\
\hline Lennartsson [24] & 2015 & 56 & $H / B$ & $\begin{array}{l}\text { DCSRD; } \\
\text { exclusion w/ } \\
\text { SEQ }\end{array}$ & $\begin{array}{l}\text { DHEA-S; ACTH; ACTH } \\
\text { and DHEA-S post- } \\
\text { TSST (TSST stress test } \\
\text { performed) }\end{array}$ & $\begin{array}{l}\downarrow D H E A-S, \downarrow \text { post-TSST DHEA-S, } \\
\text { NI ACTH, NI post-TSST ACTH }\end{array}$ & \\
\hline Tao [23] & 2015 & 171 & $H / B$ & Maslach & MSC, ACTH & $\uparrow A C T H, \uparrow M S C$ & \\
\hline Jonsson [29] & 2015 & 51 & $\mathrm{H} / \mathrm{B}$ & SMBQ & MSC, MST & NI MSC, $\downarrow$ MST & \\
\hline Lennartsson [27] & 2015 & 56 & $H / B$ & $\begin{array}{l}\text { DCSRD and } \\
\text { SMBQ }\end{array}$ & $\begin{array}{l}\text { MSC, MST, ACTH, } \\
\text { ACTH post-TSST (TSST } \\
\text { stress test performed) }\end{array}$ & $\begin{array}{l}\text { NI MSC, NI MST, NI ACTH, NI } \\
\text { post-TSST ACTH }\end{array}$ & $\begin{array}{l}\text { Severe BO: lower ACTH, cortisol response to TSST vs } \\
\text { controls, whereas low BO: higher ACTH, cortisol responses } \\
\text { vs controls }\end{array}$ \\
\hline Schmaling [26] & 2015 & 62 & Healthy & ADAS & $A \cup C, S C R$ & $\downarrow A \cup C, \downarrow S C R$ & $\begin{array}{l}31 \text { couples studied, one of which had chronic fatigue (but } \\
\text { not (FS) }\end{array}$ \\
\hline Sveinsdottir [68] & 2015 & 1150 & $\begin{array}{l}\text { Chronic } \\
\text { Lombalgia }\end{array}$ & CFQ & DAC, CAR, SCR, NSC & NI DAC, NI CAR, NI NSC, NI SCR & \\
\hline Powell [67] & 2015 & 76 & $\begin{array}{l}\text { Multiple } \\
\text { Sclerosis }\end{array}$ & CFQ & DAC, CAR, SCR & $\downarrow D A C, \uparrow C A R, N I S C R$ & $\begin{array}{l}\text { Sleep disorders excluded; adjusted for depressive } \\
\text { symptoms; NSC not published. Multiple sclerosis had } \\
\text { increased awakening cortisol and decreased CAR }\end{array}$ \\
\hline Cruz [70] & 2015 & 43 & $\begin{array}{l}\text { Breast } \\
\text { cancer }\end{array}$ & $\mathrm{BFI}$ and CFQ & MSC, DHEA-S & NI MSC, NI DHEA-S & Pts undergoing ChTx included anthracyclines \\
\hline Marchand [31] & 2014 & 1043 & $H / B$ & Maslach & DAC, CAR, SCR, NSC & $\uparrow D A C, \downarrow C A R, \uparrow S C R, \downarrow N S C$ & $\begin{array}{l}\text { Only BO; Groups of severe distress or depression not } \\
\text { included }\end{array}$ \\
\hline Aggarwal [30] & 2014 & 227 & Healthy & CFQ & $\begin{array}{l}\text { MSC, NSC, } 0.25 \mathrm{mg} \\
\text { DST, DHEA-S }\end{array}$ & $\begin{array}{l}\text { NI MSC, NI NSC, NI 0.25mg DST, } \\
\downarrow \text { DHEA-S }\end{array}$ & $\begin{array}{l}\text { Evaluation of chronic, widespread pain, chronic orofacial } \\
\text { pain, chronic fatigue (but not (FS), irritable bowel } \\
\text { syndrome }\end{array}$ \\
\hline
\end{tabular}


Table 1 Summary of selected studies (Continued)

\begin{tabular}{|c|c|c|c|c|c|c|c|}
\hline Tell D [71] & 2014 & 130 & $\begin{array}{l}\text { Breast } \\
\text { cancer }\end{array}$ & $\mathrm{MFI}$ & DAC, CAR, SCR, NSC & $\downarrow C A R, \downarrow S C R, \uparrow D A C, \uparrow N S C$ & $\begin{array}{l}\text { Post-surgery breast cancer, regardless of ChTx. Not } \\
\text { adjusted to sleeping patterns }\end{array}$ \\
\hline Wolfram [32] & 2013 & 53 & $H / B$ & Maslach & $\begin{array}{l}\text { MSC, } 1 \mathrm{mcg} \text { CST, DST/ } \\
\text { CRH test (1.5mg DST } \\
+100 \mathrm{mcg} \text { CRH) }\end{array}$ & $\begin{array}{l}\text { NI MSC, } \downarrow \text { post- } 1 \mathrm{mcg} \text { ACTH, Nl } \\
\text { ACTH and cortisol DST-CRH }\end{array}$ & $\begin{array}{l}\text { High over-commitment present blunted serum and salivary } \\
\text { cortisol and ACTH responses to DST-CRH test }\end{array}$ \\
\hline Klaassen [33] & 2013 & 27 & Healthy & $\begin{array}{l}\text { MP (Beck \& } \\
\text { Luine, 2010) and } \\
\text { Stress Tasks } \\
\text { (Wang, 2006) }\end{array}$ & MSC, post-stress & NI MSC, NI post-stress & $\begin{array}{l}\text { A complex test sequence was performed but not } \\
\text { reproduced }\end{array}$ \\
\hline Eek [34] & 2012 & 581 & Healthy & SOFI-20 & $\begin{array}{l}\text { DAC, CAR, NSC, SCR, } \\
\text { MSC }\end{array}$ & $\begin{array}{l}\text { NI DAC, NI CAR, NI NSC, NI SCR, } \\
\text { NI MSC }\end{array}$ & $\begin{array}{l}\text { Women: reduced awakening, increased CAR, increased SCR; } \\
\text { Men: increased awakening and reduced CAR - when } \\
\text { fatigued }\end{array}$ \\
\hline Sjors [35] & 2012 & 247 & $H / B$ & $\begin{array}{l}\text { DCSRD and } \\
\text { SMBQ }\end{array}$ & $\mathrm{DAC}, 15 \mathrm{~min}$ CAR & $\mathrm{NI} D A C, \mathrm{Nl} 15 \mathrm{~min}$ CAR & \\
\hline Rahman [54] & 2011 & 30 & CFS & $\begin{array}{l}\text { Previous Dx - } \\
\text { No } \\
\text { questionnaire }\end{array}$ & MSC, SCR, NSC & NI MSC, NI SCR, NI NSC & \\
\hline Moya-Albiol [37] & 2010 & 64 & $H / B$ & Maslach & DAC, CAR & $\mathrm{NI} D A C, \mathrm{NI} C A R$ & \\
\hline Kumari [38] & 2009 & 4,299 & Healthy & SF-36 & DAC, CAR, NSC, SCR & $\downarrow D A C, \downarrow C A R, \uparrow N S C, \downarrow S C R$ & $\begin{array}{l}\text { Adjusted for WC*, BMI, sleep duration, CVD medication, } \\
\text { depressive symptoms, smoking, alcohol intake provides NI } \\
\text { awakening but lower SCR }\end{array}$ \\
\hline Osterberg [39] & 2009 & 304 & $H / B$ & Maslach & DAC, CAR, NSC, SCR & NI DAC, NI CAR, $\downarrow N S C, \uparrow S C R$ & 0.5mg DST was not compared to controls \\
\hline Wingenfeld [41] & 2009 & 279 & $H / B$ & $\begin{array}{l}\text { Maslach and } \\
\text { Maastricht }\end{array}$ & $A \cup C, S C R$ & $\mathrm{NI} A \cup C, \mathrm{NI} S C R$ & $\begin{array}{l}\text { DAC and CAR not done; conclusions different from results. } \\
\text { For AUC, Low BO: NI, moderate: increased, severe: } \\
\text { decreased }\end{array}$ \\
\hline Rydstedt [40] & 2009 & 76 & Healthy & NRWS & DAC, NSC & $\mathrm{NI} D A C, \mathrm{NI} N S C$ & \\
\hline Papadopoulos [55] & 2009 & 38 & CFS & CFQ and SF-36 & $\begin{array}{l}\text { MSC, AUC, morning } \\
\text { AUC, 0,5mg DST }\end{array}$ & $\begin{array}{l}\uparrow M S C, \uparrow A \cup C, \uparrow M A \cup C, \text { NI } 0.5 \mathrm{mg} \\
\text { DST }\end{array}$ & $\begin{array}{l}\text { Data on absolute cortisol levels at each point not } \\
\text { published. DST reduction evaluated by percent reduction. }\end{array}$ \\
\hline Bay [72] & 2009 & 75 & $\begin{array}{l}\text { Post } \\
\text { traumatic } \\
\text { brain injury }\end{array}$ & POMS & AUC & $\mathrm{NI} A \cup C$ & $\begin{array}{l}\text { Correlation between brain injury-related fatigue level and } \\
\text { cortisol AUC. Basal and NSC results not reported; SCR not } \\
\text { evaluated. }\end{array}$ \\
\hline Sudhaus [73] & 2009 & 43 & $\begin{array}{l}\text { Chronic } \\
\text { Lombalgia }\end{array}$ & MFI & DAC, CAR, MAUC & $\begin{array}{l}\downarrow C A R, N I D A C, N I \text { MAUC } \\
\text { (correlation between fatigue } \\
\text { levels among low back pain } \\
\text { subjects) }\end{array}$ & $\begin{array}{l}\text { ** Colocar como SAUDÁVEL - porque é só lombalgia (Low } \\
\text { back pain had increased CAR than controls). }\end{array}$ \\
\hline Lindeberg [36] & 2008 & 78 & Healthy & SF-36 & DAC, CAR, NSC, SCR & NI DAC, $\downarrow C A R, N I N S C, \downarrow S C R$ & \\
\hline Sertoz [42] & 2008 & 72 & $\mathrm{H} / \mathrm{B}$ & Maslach & $\begin{array}{l}\text { Basal and post } \\
1.0 m c g \text { DST cortisol }\end{array}$ & $\mathrm{NI}$ basal cortisol and 1.0mg DST & \\
\hline Bellingrath [43] & 2008 & 101 & $H / B$ & $\begin{array}{l}\text { Maslach and } \\
\text { Maastricht }\end{array}$ & $\begin{array}{l}\text { DAC, CAR, NSC, SCR, } \\
0.25 \mathrm{mg} \text { DST }\end{array}$ & $\begin{array}{l}\mathrm{NI} \text { DAC, NI CAR, NI NSC, NI SCR, } \\
\downarrow 0.25 \mathrm{mg} \text { DST }\end{array}$ & \\
\hline Nater [57] & 2008 & 185 & CFS & SF-36 and MFI & DAC, CAR, MAUC & NI DAC, NI CAR, \MAUC & \\
\hline
\end{tabular}


Table 1 Summary of selected studies (Continued)

\begin{tabular}{|c|c|c|c|c|c|c|c|}
\hline Torres-Harding [56] & 2008 & 108 & CFS & FSE & $A \cup C, S C R$ & $\mathrm{NI} A \cup C, \mathrm{NI} S C R$ & $\begin{array}{l}\text { Multiple psychological tests performed. Data on NSC, basal } \\
\text { and CAR not published. }\end{array}$ \\
\hline Sonnenschein [45] & 2007 & 42 & $H / B$ & Maslach & $\begin{array}{l}\text { CAR, } 0.5 \mathrm{mg} \text { DST, } \\
\text { DHEA-S }\end{array}$ & $\begin{array}{l}\mathrm{NI} \text { CAR, NI } 0.5 \mathrm{mg} \text { DST, NI } \\
\text { DHEA-S }\end{array}$ & $\begin{array}{l}\text { Adjusted for depression, sleep quality. Awakening levels } \\
\text { and each level graphics not available }\end{array}$ \\
\hline Harris [44] & 2007 & 44 & Healthy & SF-36 & DAC, CAR, NSC, SCR & NI DAC, NI CAR, NI NSC, NI SCR & $\begin{array}{l}\text { Other aspects also correlated: complains, job stress and } \\
\text { demand, QOL and coping. Adjusted for coffee and } \\
\text { tobacco. }\end{array}$ \\
\hline Langelaan [46] & 2006 & 55 & $H / B$ & Maslach & $\begin{array}{l}\text { DAC, CAR, } 0.5 \mathrm{mg} \text { DST, } \\
\text { DHEA-S }\end{array}$ & $\begin{array}{l}\text { NI DAC, NI CAR, NI 0.5mg DST, } \\
\text { NI DHEA-S }\end{array}$ & $\begin{array}{l}\text { Engaged work also compared and had stronger } \\
\text { suppression in DST }\end{array}$ \\
\hline Mommersteeg [47] & 2006 & 109 & Healthy & NC-WHO & $\begin{array}{l}\text { DAC, CAR, } 0.5 \mathrm{mg} \text { DST, } \\
\text { SCR, AUC, NSC }\end{array}$ & $\begin{array}{l}\mathrm{NI} \text { DAC, NI CAR, NI NSC, NI SCR, } \\
\text { NI 0.5mg DST, NI AUC }\end{array}$ & \\
\hline Barroso [74] & 2006 & 40 & HIV & HRFS & MSC, NSC & $\downarrow M S C, \uparrow N S C$ & \\
\hline Jerjes [58] & 2006 & 80 & CFS & CFQ & UFC, TCM & $\downarrow U F C, N I T C M$ & \\
\hline Grossi [48] & 2005 & 64 & $H / B$ & SMBQ & DAC, CAR & $\downarrow D A C, \uparrow C A R$ & $\begin{array}{l}\text { Groups were high } \times \text { moderate } \times \text { low } \mathrm{BO} \text { score; correlation } \\
\text { was significant }\end{array}$ \\
\hline Segal [59] & 2005 & 40 & CFS & $\begin{array}{l}\text { No } \\
\text { questionnaire }\end{array}$ & MSC, $1 \mathrm{mcg}$ CST & $\downarrow$ MSC, $\downarrow 1 \mathrm{mcg}$ CST & DHEA-S collected only in CFS. No questionnaires used. \\
\hline Jerjes [60] & 2005 & 35 & CFS & CFQ & MSC, SCR, NSC, AUC & $\downarrow M S C, \downarrow S C R, \downarrow A \cup C \downarrow, N I N S C$ & \\
\hline Bower [75] & 2005 & 29 & $\begin{array}{l}\text { Breast } \\
\text { cancer }\end{array}$ & SF-36 & DAC, AUC, SCR, NSC & $\uparrow A \cup C, \downarrow S C R, N I D A C, \uparrow N S C$ & $\begin{array}{l}\text { Post-ChTx (regardless of time) complete cancer remission } \\
\text { and exclusion of other disorders }\end{array}$ \\
\hline McLean [76] & 2005 & 55 & $\begin{array}{l}\text { Fybro- } \\
\text { mialgia }\end{array}$ & SF-36 & $\begin{array}{l}\text { DAC, } 60 \mathrm{~min} \text { CAR, SCR, } \\
\text { AUC, NSC }\end{array}$ & $\begin{array}{l}\text { NI DAC, NI 60min CAR, NI SCR, } \\
\text { NI AUC, NI NSC (correlation } \\
\text { between fatigue levels among } \\
\text { FMG subjects) }\end{array}$ & FMG subjects presented NI DAC and CAR, as controls. \\
\hline Roberts [62] & 2004 & 92 & CFS & CFQ and SF-36 & DAC, CAR, MAUC & NI DAC, $\downarrow C A R, \downarrow M A \cup C$ & \\
\hline Crofford [61] & 2004 & 72 & CFS/ FMG & POMS & $\begin{array}{l}\text { ACTH, MSC, SCR, NSC, } \\
\text { AUC }\end{array}$ & $\begin{array}{l}\mathrm{NI} A C T H, \mathrm{NI} S C R, \mathrm{NI} N S C, \downarrow A \cup C \\
\mathrm{NI} \text { MSC }\end{array}$ & $\begin{array}{l}\text { Tests performed in: CFS, FMG and CFS + FMG; FMG w/o } \\
\text { fatigue had NI AUC and increased BMC levels }\end{array}$ \\
\hline Moch [50] & 2003 & 16 & $H / B$ & Maslach & $\begin{array}{l}\text { UFC, DHEA-S, ACTH, } \\
\text { MSC }\end{array}$ & $\begin{array}{l}\text { \UFC, NI DHEA-S, NI ACTH, } \\
\downarrow M S C\end{array}$ & Only women; longitudinal evaluation - NI initial cortisol. \\
\hline De Vente [49] & 2003 & 45 & $H / B$ & Maslach & DAC, MSC, post-TSST & $\uparrow D A C, \uparrow M S C$, NI post-TSST & \\
\hline Gaab [63] & 2002 & 42 & CFS & $\mathrm{MFI}$ & $\begin{array}{l}\text { DAC, CAR, SCR, } 0.5 \mathrm{mg} \\
\text { DST }\end{array}$ & $\begin{array}{l}\downarrow 0.5 \mathrm{mg} \text { DST, NI AUC, NI CAR, NI } \\
\text { DAC, NI SCR, NI NSC }\end{array}$ & CAR also performed at 15,45 and $60 \mathrm{~min}$. \\
\hline Dekkers [77] & 2000 & 53 & $\begin{array}{l}\text { Rheumatoid } \\
\text { Arthritis }\end{array}$ & MFI & DAC, CAR, SCR, AUC & $\mathrm{NI} A \cup C, \mathrm{NI} S C R \mathrm{NI}, \downarrow D A C, \uparrow C A R$ & $\begin{array}{l}5 / 25 \text { subjects with RA taking prednisone }(5-10 \mathrm{mg} / \mathrm{d}) \text {; RS } \\
\text { subjects had smaller SCR, increased AM cortisol and } \\
\text { decreased CAR. } 15 \text { and } 45 \text { min CAR also performed. }\end{array}$ \\
\hline Melamed [51] & 1999 & 111 & $H / B$ & $\begin{array}{l}\text { SMBQ and } \\
\text { Maastricht }\end{array}$ & MSC and 4 PM cortisol & $\uparrow M S C$, NI 4PM cortisol & \\
\hline Pruessner [52] & 1999 & 66 & Healthy & Maslach & DAC, CAR, 0.5mg DST & $\downarrow D A C, \downarrow C A R, \downarrow 0.5 \mathrm{mg}$ DST & $15 \mathrm{~min}$ and $60 \mathrm{~min}$ CAR also performed \\
\hline Strickland [65] & 1998 & 74 & CFS & $\begin{array}{l}\text { Not specified/ } \\
\text { detailed }\end{array}$ & MSC, NSC & $\downarrow N S C$, NI MSC & Adjusted for depression \\
\hline
\end{tabular}


Table 1 Summary of selected studies (Continued)

\begin{tabular}{|c|c|c|c|c|c|c|c|}
\hline Young [66] & 1998 & 45 & CFS & $\mathrm{NC}-\mathrm{WHO}$ & UFC, SCR, MSC, AUC & NI UFC, NI SCR, NI MSC, NI AUC & \\
\hline Scott [64] & 1998 & 28 & CFS & $\begin{array}{l}\text { Not specified } \\
\text { (not detailed) }\end{array}$ & $\begin{array}{l}\text { MSC, ACTH, } 100 \mathrm{mcg} \\
\text { CRH cortisol } \\
\text { stimulation }\end{array}$ & $\begin{array}{l}\text { NI MSC, NI ACTH, CRH stim test: } \\
\downarrow \text { cortisol, } \downarrow \text { ACTH }\end{array}$ & \\
\hline Raikkonen [53] & 1996 & 22 & Healthy & Not assessed & $\begin{array}{l}1 \text { mcg CST, DST (non } \\
\text { specified), OGTT, MSC, } \\
\text { ACTH, cortisol/ACTH } \\
\text { ratio }\end{array}$ & $\begin{array}{l}\uparrow \text { Cortisol/ACTH ratio; } \uparrow C S T, N I \\
\text { DST, NI OGTT, NI MSC, NI ACTH }\end{array}$ & $\begin{array}{l}\text { Full article not assessed - not in PUBMED or other } \\
\text { database }\end{array}$ \\
\hline
\end{tabular}

Questionnaires: SMBQ Shirom-Melamed Burnout Questionnaire, BFI Brief Fatigue Inventory, CFQ Chalder Fatigue Questionnaire, Maslach Maslach Burnout Inventory, SF-36 Short Form Health Survey 36, NC-WHO Neurasthenia Criteria, DCSRD Diagnosis Criteria of Stress-related Exhaustion Disorder, SEQ Stress-Energy Questionnaire, ADAS Abbreviated Dyadic Adjustment Scale, MFI Multidimensional Fatigue Inventory, FAQ Fatigue Assessment Questionnaire, MP Memory Performance, POMS Profile of Mood States, Stress Tasks; FSE Fatigue Severity Scale, SOFI Swedish Occupational Fatigue Inventory, Maastricht Maastricht Vital Exhaustion Questionnaire, NRWS Need for Recovery from Work Scale, HRFS HIV-Related Fatigue Scale

Other abbreviations: CFS Chronic Fatigue Syndrome, H/B Healthy/Burnout, 24h-UFC 24-h Urinary Free Cortisol, FMG Fibromyalgia, $\uparrow:$ Increased or elevated, $\downarrow:$ Decreased or reduced, $\rightarrow$ : Unchanged, NI Normal 